\title{
Étude expérimentale du couplage hydromécanique de joints rocheux
}

\section{SIBAI}

M. HAJI SOTOUDEH

Laboratoire de mécanique de Lille (URA-CNRS 1441),

boulevard Paul-Langevin,

59655 Villeneuve-d'Ascq

Cedex

\section{J.-P. HENRY}

École des Mines d'Alès, 6, avenue de Clavières, 30319 Alès Cedex
Dans cet article, on présente tout d'abord le dispositif expérimental conçu pour l'étude des comportements mécanique et hydromécanique de joints isolés, très fermés et sous sollicitations normales. L'avantage de ce dispositif par rapport aux montages existants est de permettre de quantifier l'évolution et la distribution de la pression interstitielle dans le joint. On présente ensuite les résultats expérimentaux obtenus sur deux types de joints rocheux : un granite et un schiste. Les résultats ont permis de déterminer : l'évolution de l'épaisseur de joints en fonction de la contrainte effective ; les forces appliquées sur les surfaces des joints durant l'écoulement; l'influence de l'écoulement sur l'ouverture du joint.

\section{Experimental study of hydromechanical behavior of rock joint}

In this paper, an experimental device is presented for the study of the mechanical and the hydromechanical behavior of an isolated and closed joint under normal stress. Comparing with other existing devices, it permits the measurement of pore pressure within the joint, which is a new advance in this topic. And then, experimental results are presented on two types of rock joints : granit and schist. The evolution of the joint's aperture, the applied forces on the surfaces of joint during the flow and the influence of flow on joint aperture are presented. 


\section{1}

\section{Introduction}

Un massif rocheux est généralement constitué d'une matrice plus ou moins poreuse et d'un réseau de fractures. Ces discontinuités, exprimées à l'échelle de l'ouvrage considéré, ont une influence prépondérante sur les caractéristiques du massif c'est-à-dire sur ses comportements mécanique et hydraulique. Pour pouvoir étudier le comportement global d'un massif, il faut donc étudier le comportement du réseau de fractures, notamment lorsque la matrice est faiblement poreuse $[1,3]$.

Deux approches sont possibles pour la modélisation du comportement hydromécanique des massifs fracturés : la première est de considérer le massif comme un milieu poreux « homogénéisé $»$ [2], la deuxième considère le réseau de fractures comme un réseau de conduites avec des parois parallèles. Une comparaison entre ces approches a été présentée par Braester [4] et [9]. Cette étude se place dans la seconde approche. On examine ici le comportement hydromécanique d'un joint isolé en se restreignant aux joints non remplis et très fermés et en se limitant aux sollicitations normales à ce joint.

Les modèles de couplage hydromécanique sont actuellement bien développés du point de vue théorique, en milieu poreux continu avec des lois de comportement plus ou moins complexe de la partie squelette. En revanche, en ce qui concerne les milieux fissurés ou fracturés, les modèles de couplage sont encore d'un usage très limité et pour la plupart très récents [6]. Souvent, il ne s'agit pas de modèle de couplage mais de modèles hydrauliques prenant en compte la variation " d'ouverture du joint » en fonction des contraintes appliquées [5]. Les raisons de la faiblesse des modèles hydromécaniques en milieu fracturé relèvent, d'une part, des très grandes difficultés de description des discontinuités et, d'autre part, de la mauvaise connaissance des phénomènes hydromécaniques et hydrauliques dans les fractures individuelles. Dans ce travail à caractère expérimental, on se propose de développer et d'améliorer une méthode de détermination des paramètres nécessaires pour modéliser le comportement mécanique et hydromécanique d'un joint isolé.

\section{2}

\section{Préparation des éprouvettes et dispositif expérimental}

\section{1}

\section{Principe}

On a choisi de travailler sous chargement hydrostatique, c'est-à-dire d'étudier l'influence de la raideur normale sur l'écoulement. Le chargement hydrostatique permet de connaître très précisément les conditions aux limites, et de s'affranchir de l'interaction entre le montage et l'échantillon, difficulté rencontré par exemple dans les boîtes de cisaillement.

Également, on n'a pas pris le parti d'utiliser un écoulement radial ou pseudo radial à partir d'un point de source, car, d'une part, ce type d'écoulement ne représente pas la réalité et, d'autre part, il ne permet pas d'étudier correctement le couplage entre comportement mécanique (matrice + joint) et écoulement, comme on va le montrer schématiquement. Supposons, tout d'abord, un écoulement parallèle et un joint plan. Sous l'action d'une pression, le joint peut s'ouvrir légèrement et changer les caractéristiques d'épaisseur moyenne, ce qui va modifier la distribution de pression par rapport à un profil de pression à ouverture constante. Dans le cas d'un écoulement radial, le soulèvement dû à la pression est très faible, de part la « rigidité ») plus grande du bloc par rapport à l'écoulement : par conséquent, nous sommes plus proches d'un écoulement à épaisseur constante, ce qui n'est pas le cas dans la réalité [7] et [8].

Bien entendu, ce couplage, dù à la variation d'ouverture, doit être estimé par le montage envisagé. Il faudra donc mesurer des variations d'épaisseur du joint dues à l'écoulement. On aboutit donc au principe de montage de la figure 1, qui permet :

- la mesure des variations d'épaisseur de joint en trois endroits sur le profil de l'écoulement $\left(\Delta \mathrm{L}_{1}, \Delta \mathrm{L}_{2}, \Delta \mathrm{L}_{3}\right)$; - la mesure de pression en deux endroits du joint, ce qui donne quatre points de pression : $\mathrm{P}_{1}$ à l'injection, $\mathrm{P}_{2}$ et $\mathrm{P}_{3}$ dans le joint et $\mathrm{P}_{4}$ à la sortie.

\section{2}

\section{Échantillon et mesure des pressions $P_{2}$ et $P_{3}$}

La taille d'échantillon est de $65 \mathrm{~mm}$ de diamètre et est de 100 à $120 \mathrm{~mm}$ de hauteur. Pour mesurer la pression dans le joint, on a utilisé la technique classique de mesure de pression d'arrêt (tube Pitot). Pour ce faire, un trou de $3 \mathrm{~mm}$ a été foré jusqu'au plan de joint, puis on a collé à l'intérieur de ce petit forage un tube métallique qui est ensuite relié à un capteur de pression (Fig. 1).

La première mesure de pression $\left(\mathrm{P}_{2}\right)$ s'effectue à $35 \mathrm{~mm}$ de l'embase d'injection, et la seconde $\left(\mathrm{P}_{3}\right)$ à $70 \mathrm{~mm}$ de l'embase d'injection.

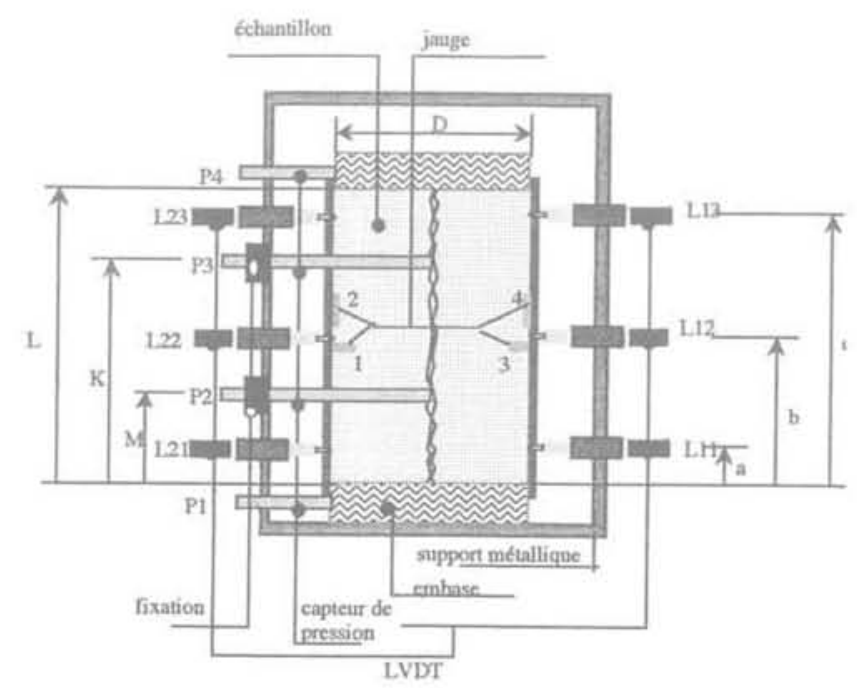

FiG. 1 Équipement de I'éprouvette $(D=64$, $\mathrm{L}=113,5, \mathrm{~K}=69,6, \mathrm{M}=35, \mathrm{a}=12, \mathrm{~b}=52$, $\mathrm{c}=92$ en $\mathrm{mm}$ ).

Sample and its Equipements $(\mathrm{D}=64, \mathrm{~L}=113,5$, $\mathrm{K}=69,6, \mathrm{M}=35, \mathrm{a}=12, \mathrm{~b}=52, \mathrm{c}=92$ in $\mathrm{mm}$ ). 


\section{Mesure de l'écrasement des joints}

Pour pouvoir mesurer des écrasements, il faut tout d'abord une base fixe. L'éprouvette est donc placée dans un cadre fixe, servant de base de mesure. L'accrochage de l'éprouvette au cadre s'effectue par l'intermédiaire des tubes mesurant les pressions $\mathrm{P}_{1}$ et $\mathrm{P}_{2}$. Les déplacements globaux sont alors mesurés par six capteurs de déplacement de type LVDT, fixés dans le cadre et placés respectivement à $35\left(\mathrm{~L}_{3}\right), 55\left(\mathrm{~L}_{3}\right)$ et $85\left(\mathrm{~L}_{3}\right) \mathrm{mm}$ de l'embase d'injection; les LVDT de chaque paire sont montés en opposition. Les capteurs utilisés sont de type RDP D5 de course $10 \mathrm{~mm}$. Si l'on fait varier la pression de confinement (mesure de raideur de joint), l'écrasement du joint est égal à la variation lue par les capteurs de déplacement, moins la déformation matricielle de l'échantillon. Cette dernière peut être enregistrée par des jauges d'extensométrie collées sur l'échantillon, mais le plus loin possible du joint, pour ne pas être perturbées par celui-ci.

\section{4}

\section{Dispositif expérimental global}

Avant de disposer l'échantillon dans le cadre, il est gainé par une membrane en silastène. Pour éviter la ren-

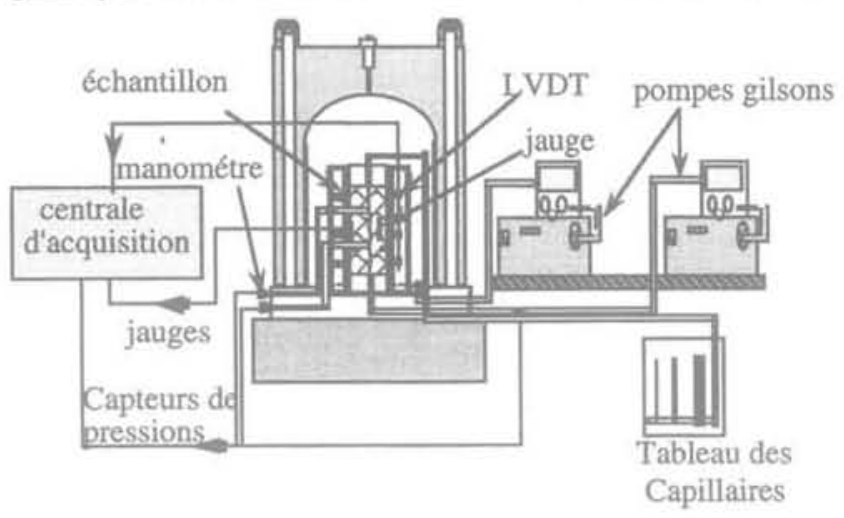

FG. 2 Représentation schématique du dispositif expérimental.

Composition of experimental apparatus. trée de la gaine dans le joint sous l'effet de la pression, ce qui pourrait la percer, le joint est, au préalable, légèrement enduit de silicone sur les faces de l'échantillon.

L'ensemble (cadre + échantillon), est placé dans une enceinte haute pression (200 MPa) de grande taille (diamètre intérieur de $250 \mathrm{~mm}$, hauteur intérieure de $350 \mathrm{~mm}$ ).

Le dispositif expérimental global, représenté par la figure 2, comprend, outre la cellule et l'échantillon décrit précédemment :

- deux pompes Gilson équipées par des modules manométriques pour la régulation de la pression de confinement $(\mathrm{Pc})$ et la pression d'injection $\left(\mathrm{P}_{1}\right)$;

- une centrale d'acquisition de données Vishay 4000 avec des tiroirs d'extensométrie et de capteurs LVDT; - un tableau de capillaires calibrés de différents diamètres (1, 3, 5 et $10 \mathrm{~mm})$, est utilisé pour la mesure du volume de fluide injecté ou expulsé ;

- deux capteurs de pressions (pour $\mathrm{P}_{2}$ et $\mathrm{P}_{3}$ ) montés sur l'embase inférieure de la cellule.

Cet équipement permet d'évaluer :

- la variation d'ouverture-fermeture du joint :

- l'évolution du champ de contrainte dans la matrice ; - la courbe de perte de charge hydraulique (évolution et distribution de la pression du fluide);

- la variation du débit en fonction de la pression d'injection (charge hydraulique) et du chargement normal du joint (pression du confinement).

\section{3}

\section{Résultats expérimentaux}

Pour cette étude, on a travaillé sur des joints frais de granite et de schiste. Les joints ont été obtenus par fendage (essai brésilien), en faisant très attention à ne pas les détériorer. Des relevés de profils pour les deux faces du joint ont été effectués au laser (au laboratoire de géomécanique de Nancy), la représentation 3D des surfaces testées est donnée respectivement sur les figures 3 et 4 pour le joint de granite et le joint de schiste. On observe une différence qualitative entre les deux morphologies, cette différence provient de la différence de rugosité entre les deux joints. Il existe plu-

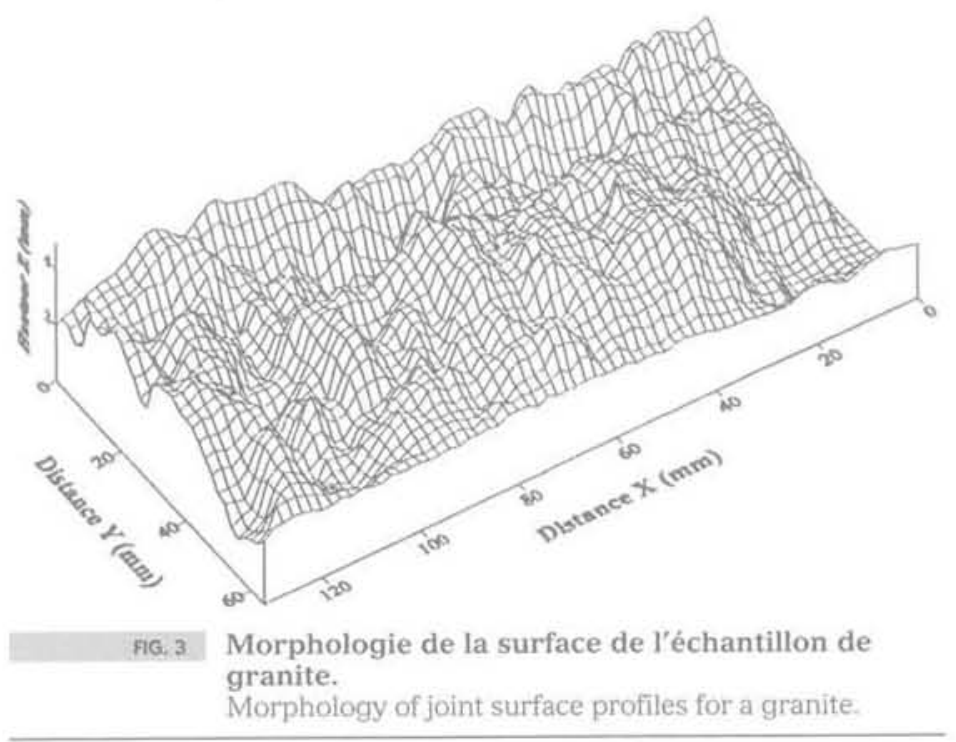




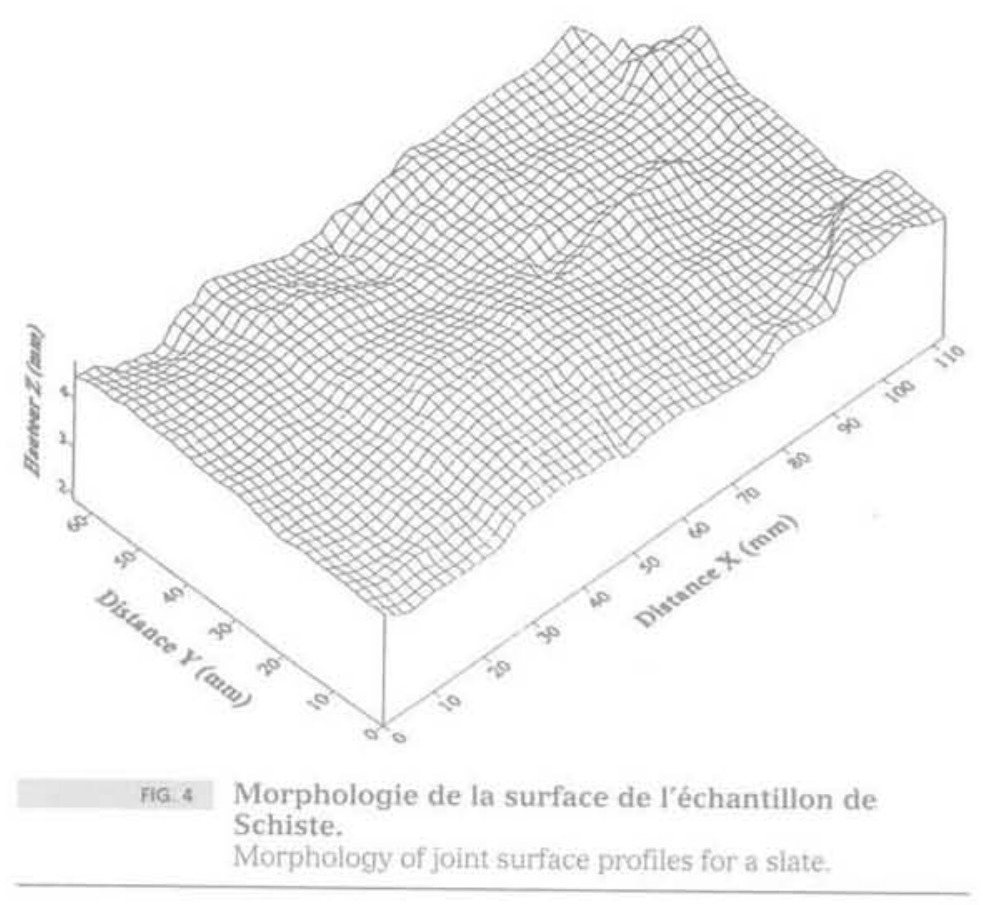

sieurs méthodes pour l'étude de la morphologie et la détermination de l'indice de rugosité. Ces méthodes plus élaborées n'ont pas été mise en cuvre, compte tenu de l'objectif orienté vers la détermination des propriétés mécaniques, hydromécaniques et non vers l'obtention d'informations morphologiques.

Trois types d'essais ont été réalisés : des essais sans pression interstitielle, des essais non drainés à pression interstitielle imposée et des essais drainés. Le premier et le deuxième type sont utilisés pour l'étude du comportement mécanique et du couplage hydromécanique. Le troisième type d'essai est utilisé pour l'étude du comportement hydromécanique.

\section{1}

\section{Comportement mécanique de joints}

L'échantillon fracturé et instrumenté, tel que décrit dans le paragraphe 2, est testé sans pression interstitielle $(\mathrm{Pi}=0)$, uniquement sous la pression de confinement. Les figures 5 et 6 (pour le granite et le schiste respectivement) représentent les déplacements corrigés et enregistrés par les six capteurs opposés deux à deux en fonction de la pression de confinement $(\mathrm{Pc})$ au cours des cycles de chargement-déchargement. Ces mesures soulèvent quelques remarques:

1) les trois déplacements sont très différents les uns des autres, particulièrement pour les pressions de confinement entre 0 et $1 \mathrm{MPa}$. Les capteurs détectent donc convenablement les modifications locales apportées par le joint;

2) d'après les cycles de chargement-déchargement on observe l'existence de boucles d'hystérésis et on s'aperçoit qu'il n'y a pas d'adaptation. Au contraire, le joint se met en place et des écrasements irréversibles ont lieu; l'ensemble de ces phénomènes ainsi que le frottement et la variation entre les lèvres de joint font que les courbes de chargement-déchargement varient avec les cycles.

Les moyennes des déplacements (L) (après sous- traction des déplacements dus à la matrice et au montage) sont présentés sur la figure 5 pour le joint de granite et sur la figure 6 pour le joint de schiste. On remarquera qu'après une pression de confinement de $15 \mathrm{MPa}$ pour le joint de granite et de $10 \mathrm{MPa}$ pour le joint de schiste, les joints semblent complètement fermés.

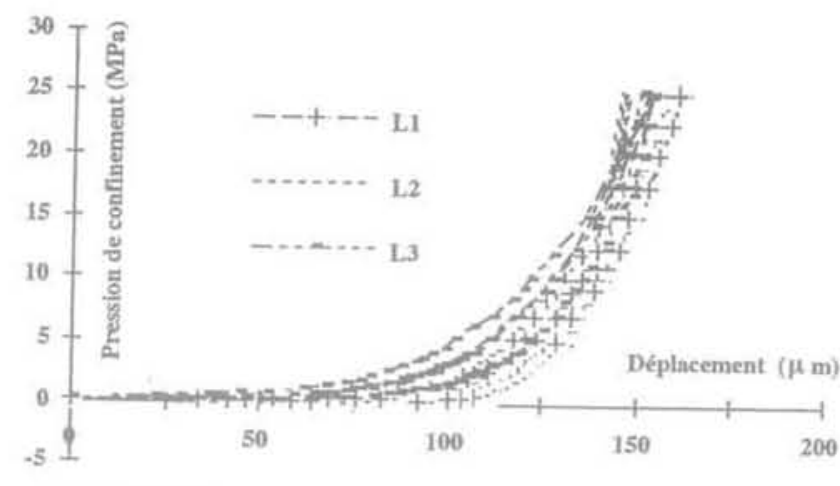

FG. 5 Déplacements enregistrés par les LVDT au cours de chargement-déchargement en contrainte hydrostatique (granite). Relation between displacement and hydostatic stress (granite).

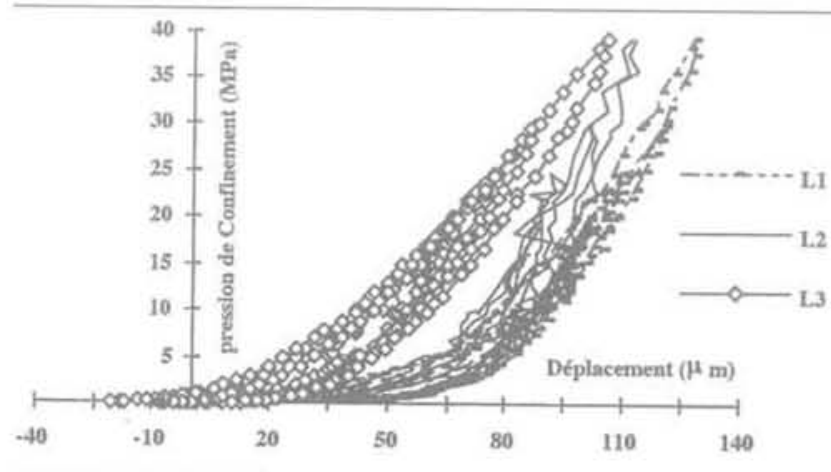

FGG.6 Déplacements enregistrés par les LVDT au cours de chargement-déchargement en contrainte hydrostatique (schiste). Relation between displacement and hydostatic stress (slate). 


\section{Essai de percolation}

Des essais de percolation préliminaires ont été réalisés pour tester l'efficacité du montage et pour répondre à quelques questions élémentaires :

(a) du point de vue expérimental : si l'on effectue une variation rapide de pression d'injection, est-on capable de distinguer les phases d'écoulement transitoire et stationnaire?

(b) pour une pression de confinement donnée, existet-il une pression d'injection qui conduit à une ouverture (ou soulèvement) du joint?

Pour répondre à ces premières interrogations, de nombreux tests d'injection ont été effectués pour différentes pressions de confinement. Dans cette série d'essais, une seule prise de pression, à 0,39 de la hauteur de l'échantillon à partir du point d'injection, a été installée. La figure 7 montre un résultat typique de ces essais sous une pression de confinement de $15 \mathrm{MPa}$. La pression d'injection est augmentée par paliers, puis déchargée également par paliers.

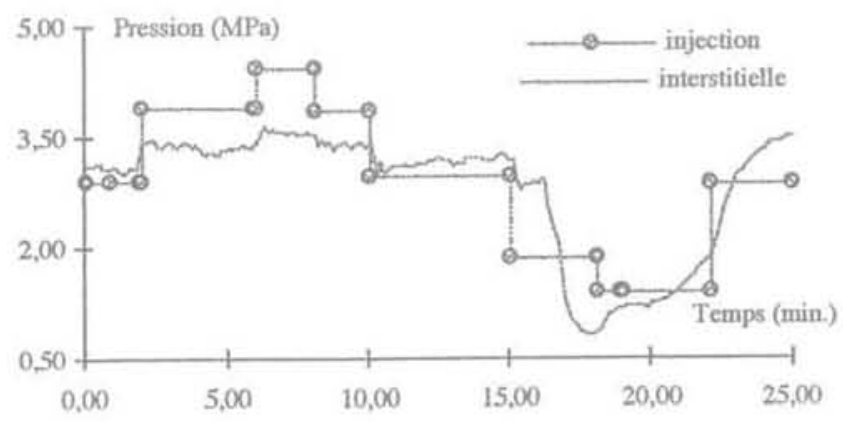

FG.7 Exemple de réponse de pression dans le joint en fonction de variations de pression d'injection à $\mathrm{Pc}=15 \mathrm{MPa}$ (granite).

The pressure reponse inside of joint in fonction of different injection pressure for $\mathrm{Pc}=15 \mathrm{MPa}$ (granite).

On remarque qu'en montée ou en descente de la pression, la réponse du capteur de pression interstitielle, est quasi instantanée. Ceci signifie que l'écoulement transitoire est de très courte durée, et que la compressibilité du fluide dans les tuyaux n'a pas trop d'influence sur la mesure. La figure 7 montre aussi qu'après une montée de la pression d'injection, la pression interstitielle augmente presque instantanément : celle-ci semble décroitre légèrement par la suite, ce qui pourrait indiquer que le joint a tendance à s'ouvrir progressivement (effet du frottement).

L'ouverture du joint semble donc un paramètre important dans l'étude de l'écoulement. Cette ouverture moyenne peut être enregistrée grâce aux six capteurs. Une longue série d'essais a été effectuée en fixant tout d'abord la pression de confinement à Pc, puis en augmentant par palier la pression d'injection jusqu'à Pc-0,5 MPa, puis en la diminuant par paliers. La pression de confinement est ensuite augmentée..., tout en répétant la procédure, puis diminuée. La figure 8 montre une courbe typique lorsque la pression d'injection n'est pas trop éloignée de la pression de confinement, laquelle vaut environ $5 \mathrm{MPa}$. Dans la première phase, l'augmentation par paliers de la pression de confinement ne change aucun paramètre : débit quasi- ment nul, pas de variation d'ouverture du joint. Lorsque $\mathrm{P}_{1}$ atteint environ $4,4 \mathrm{MPa}$ la pression $\mathrm{P}_{2}$ augmente pour atteindre environ $3 \mathrm{MPa}$; le débit augmente et la jauge $J_{3}$ indique des déformations de compression.

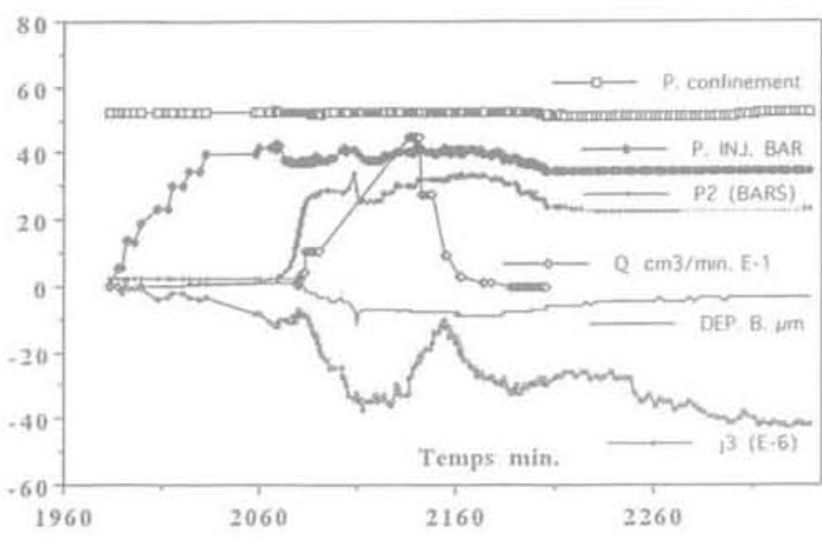

FiG. 8 Courbes de déplacement et des pressions en fonction du chargement hydrostatique pendant l'ceuvre (granite).

Typical curve of displacement and pressure during the opening of joint for $\mathrm{Pc}=5 \mathrm{MPa}$ (granite).

\section{s)ing}

\section{Comportement du couplage (contrainte normale-pression interstitielle)}

Les essais préliminaires présentés au paragraphe précédent ont montré une interaction entre la contrainte normale et la pression interstitielle en sollicitant l'ouverture du joint. En outre, la manque d'une formulation claire de la pression effective appropriée aux joints rocheux a conduit à mettre en cuvre une série d'essais spécifiques afin d'obtenir une description objective de l'interaction contrainte normale-pression interstitielle pour ces joints. Des essais non drainés à pression interstitielle imposée ont été réalisés. En effet, à pression de confinement constante, on applique par paliers une pression interstitielle uniforme sur toute la longueur du joint; la variation de la pression interstitielle induit une variation des déplacements.

La figure 9 montre une comparaison entre les essais sans et avec pression interstitielle (pour le granite). Cette figure représente les déplacements moyens corrigés en fonction de $\mathrm{Pc}-\mathrm{Pi}$. D'une manière générale, l'allure des courbes se ressemble. Mais ces courbes ne sont jamais superposables ; on constate une différence systématique. En effet, la pression interstitielle n'agit que sur une partie du joint où les épontes ne sont pas en contact. Cette pression interstitielle réduit la force appliquée sur la surface du joint. La valeur de la force opposée à la contrainte normale, due à la pression interstitielle, dépend de la surface accessible au fluide interstitiel. Le rapport entre la surface accessible au fluide et la surface totale du joint joue sûrement un rôle important dans la mesure des déplacements, d'où la différence entre les deux courbes. 


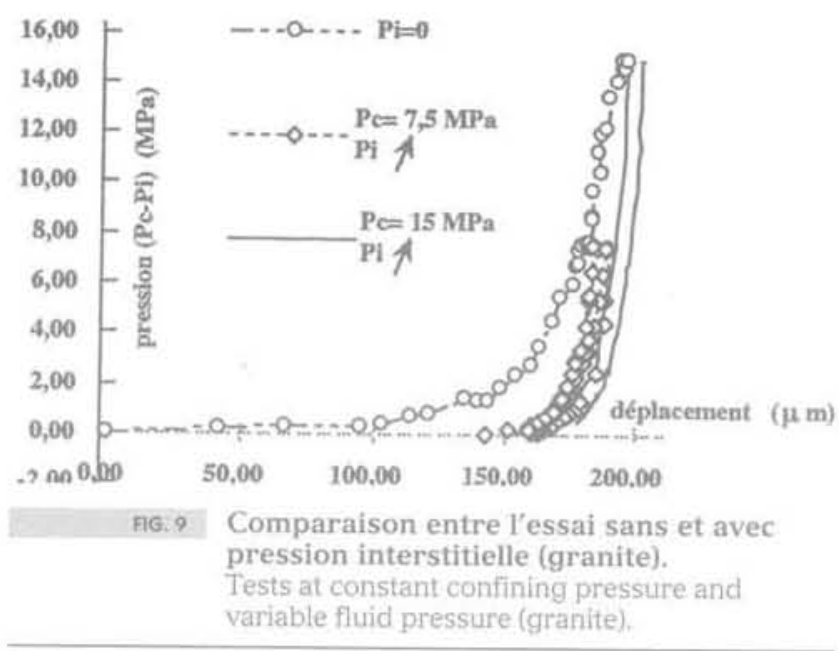

13:28

Comportement hydromécanique (écoulement dans le joint)

Ce sont des essais drainés avec mesure de la conductivité hydraulique. En effet, à différentes pressions de confinement $(1,2,4,5,7,10$ et $15 \mathrm{MPa})$, on fait des montées et puis des décharges (sur la même éprouvette) par paliers en pression d'injection. A chaque pression d'injection, on mesure la conductivité hydraulique (une fois l'écoulement stationnaire) et on enregistre les pressions interstitielles $\left(\mathrm{P}_{1}, \mathrm{P}_{2}, \mathrm{P}_{3}, \mathrm{P}_{4}\right)$. Les observations importantes sont présentées sur les figures 10,11 et 12 .

La figure 10 montre les courbes (pour le granite) de la variation du débit en fonction de la pression d'injection (pour des chemins croissants et décroissants de pression d'injection) à différentes pressions de confinement. On constate sur la figure 10 que l'augmentation de la pression de confinement induit une forte réduction du débit. On remarque aussi que, dans tous les essais, les débits du chemin décroissant de pression d'injection sont plus grands que celui du chemin croissant. Les mesures de déplacement des épontes pour un essai à $10 \mathrm{MPa}$ de pression de confinement sont représentées sur la figure 11. On remarque que $\mathrm{L}_{4}$ ne varie pas de façon significative, ni pour un chemin croissant ni pour un chemin décroissant. Le déplacement donné par $\mathrm{L}_{2}$ ne varie qu'à partir de $8 \mathrm{MPa}$ de pression d'injection et sa variation reste très limitée. Par contre L est très sensible aux variations de la pression d'injection. On constate aussi que le joint parait plus ouvert pour un chemin de pression d'injection décroissant, ce qui confirme l'irréversibilité des propriétés hydrauliques.

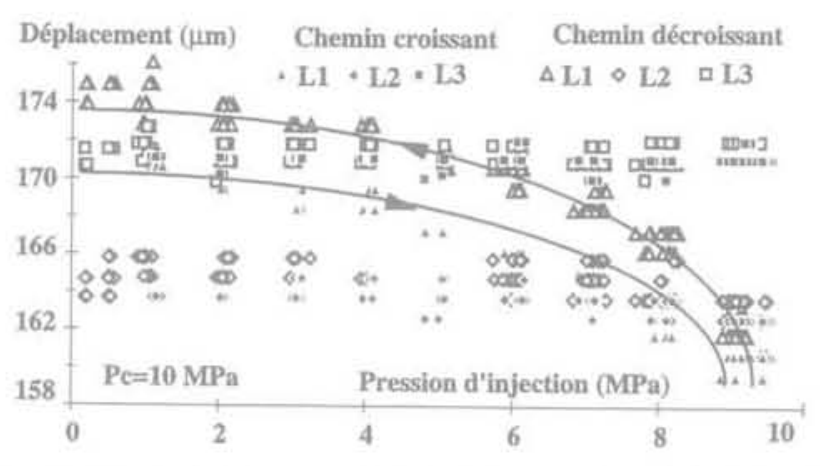

FiG. II Variation des déplacements en fonction de la pression d'injection. Displacement in fonction of injection pressure.

La figure 12 montre les profils des pressions interstitielles obtenues pendant l'essai à 7,5 MPa de pression de confinement (pour le granite). Quand la pression de drainage est nulle, on remarque que les capteurs de pression $\left(\mathrm{P}_{1}, \mathrm{P}_{2}, \mathrm{P}_{4}\right)$ donnent une distribution quasi linéaire. En revanche, le capteur $\mathrm{P}_{3}$ donne des valeurs très faibles. Pourtant quand on augmente la pression de drainage, on voit immédiatement une augmentation de $\mathrm{P}_{3}$. En conséquence, on peut supposer que les liaisons entre le front de drainage $\mathrm{P}_{4}$ et le point de mesure de $\mathrm{P}_{3}$ ont lieu par des vides de grandes dimensions (peu de résistance hydraulique). Au contraire, les liaisons entre le front d'injection $\mathrm{P}_{4}$ et le point de mesure de $\mathrm{P}_{2}$ ont de petites dimensions et/ou les liaisons entre $P_{2}$ et $P_{3}$ ne sont pas bien développées.

\section{Débit mm3/min.}

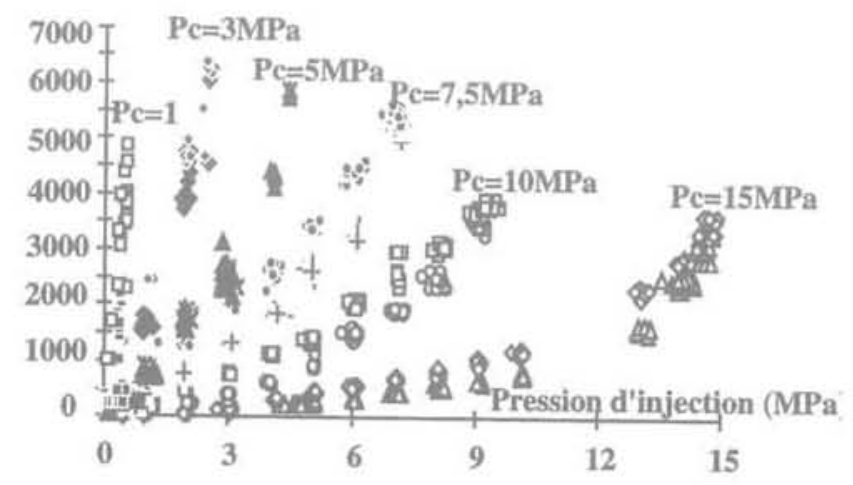

FIG. 10 Variation de débit en fonction de la pression d'injection à pression de drainage $=0$ (granite).

Conductivity hydraulic in fonction of injection pressure at drainage pressure $=0$ (granite) .

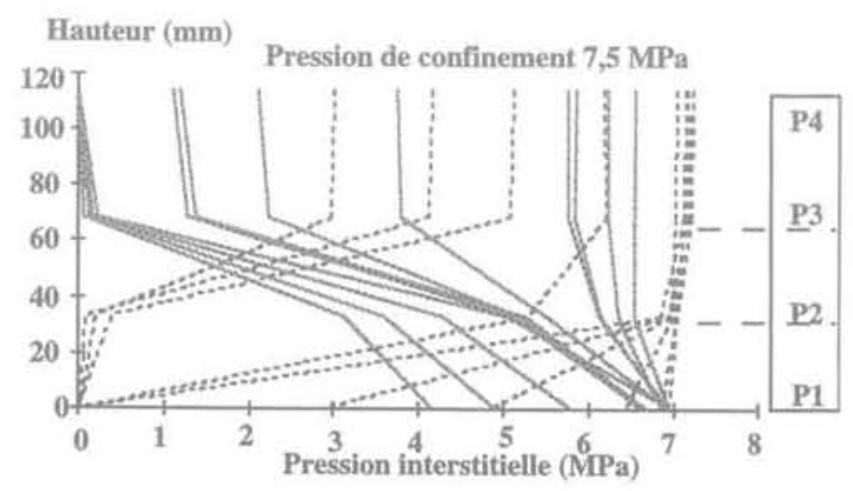




\section{Conclusion}

Cette étude méthodologique sur un nouveau montage expérimental d'étude du comportement des joints frais, avec écoulement de fluide, a permis de montrer les points suivants:

- le montage utilisé est d'une précision suffisante, et permet de suivre la pénétration du fluide dans le joint, de déterminer l'ouverture moyenne du joint et la redistribution des contraintes dans la matrice rocheuse ;

- l'importance du couplage entre la déformabilité de la matrice et la pénétration du fluide dans le joint. De ce fait, une définition appropriée de la pression effective est nécessaire afin que la courbe « pression effectivedéplacement » soit indépendante de la sollicitation : - le débit dans le joint (pour une pression de confinement donnée) ne dépend pas seulement du gradient hydraulique, mais aussi de la pression moyenne du fluide.

\section{Bibliographie}

[1] Bandis S.C. Lumsden A.C. Barton N. Fundamentals of rock joint deformation. Int I Rock Mech Min Sci and Goemech. 1983, abstr. V 20, pp. 249-268

[2] Billaux D. - Hydrogéologie des milieux fracturés. Géométrie, connectivité et comportement hydromécanique. Thèse de doctorat, École des Mines de Paris. 1990.

[3] Breaster C. Discrete and continuum approaches to solution of flow problems in fractured rocks. Proceedings of Fourth canadian/american conference on hydrology, fluid flow, heat transfer and mass transport in fractured rocks, 1988, pp. 223-229.
[4] Detournay F - Hydraulic conductivity of closed rock fractures; an experimental and analytical study. Symp. on Underground Rock Engineering, 13th Canadian Rock Mechanics Symp. Toronto, 1980, pp. $168-173$

[5] Gale J.E. - Comparison of coupled fracture deformation and fluid flow models with direct measurements of fracture pore structure and stressflow properties, 28th US Symp. on rock mech.. Newman-editors, 1987. pp. 1213-1222.

6] Gentier S. - Morphologie et comportement hydromécanique d'une fracture naturelle dans un granite sous contrainte normal. Thèse de doctorat BRGM, Orlèans, 1986.

(7) Haji Sotoudeh M. - Étude expérimentale et modélisation ciu couplage hyciromécanique de joints rocheux. Thèse de docto. rat, Lille I, 1995.

[8] Haii Sotoudeh M. Sibai M. Henry J.-P. - Experimental study of hydromechanical behavior of rock joint. M.JFR2, supplementary volume, Vienne. Austria, pp. 31-36.

[9] Goodman R.E. Method of geological engineering in discontinuous rocks. West Publishing Company, 1976 